\title{
Effects of Transition Energy on Intra-Band Photoluminescence of Zinc Oxide (ZnO) Semiconductor under Low injection Level
}

\author{
Getu Endale \\ Department of Physics, CNCS, Wolkite University, P.O.Box 007, Wolkite, Ethiopia
}

Copyright (C)2019 by authors, all rights reserved. Authors agree that this article remains permanently open access under the terms of the Creative Commons Attribution License 4.0 International License

\begin{abstract}
This paper presents the effects of the transition energies on photoluminescence intensities in Zinc Oxide compound semiconductor due to the intra-band transition of free carriers. The excitation of free carriers from the valence band to conduction band and from different localized state to the conduction band by the illumination of sufficient energy is considered. A theoretical model for minority carrier trapping is also investigated to explain the dependence of the photoluminescence on the trap energy. Variation of photoluminescence intensities along with localized state energy and transition energy is considered at different temperatures. As temperature increases the photoluminescence due to the transition of free electrons from the conduction band to the valence band, from the conduction band to the localized states and from the localized states to the valence band are increasing.
\end{abstract}

Keywords Photoluminescence, Intra-band, Photon Energy, Energy Bands.

\section{Introduction}

For different semiconductor devices, one needs materials with different parameters, like energy band-gap. Physical properties are very different among different semiconductors due to distinct characteristics of energy band-gaps and impurities. These impurities play a major role in determining the electrical and optical properties of semiconductors. Almost all of today's technology involves the use of semiconductors, with the most significant aspect being the integrated circuit (IC). One of the most commonly used techniques to investigate the properties of semiconductors is Photoluminescence (PL). PL has become a standard method for the characterization of semiconductor properties. It can be used to determine energy levels, concentration of impurities, defects and fundamental properties of semiconductors [1].

$\mathrm{ZnO}$ is a typical II-VI semiconductor material with a wide band gap of $3.37 \mathrm{eV}$, intrinsic carrier concentration $\left(<10^{6} \mathrm{~cm}^{-3}\right)$ (max n-type doping $\left(>10^{20} \mathrm{~cm}^{-3}\right)$ electrons; max p-type doping $10^{17} \mathrm{~cm}^{-3}$ holes), exciton binding energy $60 \mathrm{meV}$, electron 0.24 and hole effective mass 0.59 respectively at room temperature. In direct band gap the electron may be excited without the assistance of a phonon and can be seen as a more abrupt absorption edge in the spectra. Different materials are also classified by the magnitude of their band gap energy into one of three categories: narrow-band gap, mid band gap, and wide-band gap. There has been notable interest in the use of wide band gap semiconductors for consumer as well as defense applications [2, 3, 4, 5, 6, 7].

Photoluminescence involves the irradiation of the crystal to be characterized with photons of energy greater than the bandgap energy of that material. Photoluminescence consists of impinging relatively high frequency light onto a material, exciting atomic electrons. Subsequent relaxation may result in the production of photons that are characteristic of the crystal or defect site that emits the light. The luminescent signals detected could result from the band-to-band recombination, intrinsic crystalline defects (growth defects), dopant impurities (introduced during growth or ion implantation), or other extrinsic defect levels (because of radiation or thermal effects) [8].

Photoluminescence (PL) is the spontaneous emission of light from a material under optical excitation. PL measurement is a kind of powerful and nondestructive technique, which has been carried out on most of semiconductors. When light of sufficient energy is illuminated a material, photons are absorbed and excitations are created. These excited carriers relax and emit a photon. Then PL spectrum can be collected and analyzed. However,the absorption can happen in materials, only when the energy of photon is equal to or higher than the band gap. Therefore, we have to choose different excitation source to do the measurements according to different material with different electronic band structure. The PL peak positions reveal transition energies and the PL intensity implicates the relative rates of radiative recombination [9]. From PL measurement, we can obtain a variety of material parameters, such 
as: Band gap determination, Impurity levels and defect detection and recombination mechanisms. Usually, there are always some native defects and impurities in even pure semiconductors, which will form some localized defect levels between the band gap. These defect levels certainly participate the radiative transitions. The PL energy associated with these levels can be used to identify specific defects, and the PL intensity can be used to provide relative information concerning their concentration. The PL intensity depends on the photo-excitation and temperature, which is determined by the dominant recombination process. Thus, $\mathrm{ZnO}$ is one of the most popular semiconductors due to its large improvements in growth of high quality, single crystalline $\mathrm{ZnO}$ in both epitaxial layers. And also, since $\mathrm{ZnO}$ is from one of wide band gap semiconductors, it is applicable for the emission of photon in radiative recombination mechanism [10].

Photoluminescence process is shown in the figure 1 below. This material contains a defect level (due to an impurity) in the band gap, which has a strong tendency to capture (trap) electrons from the conduction band or holes from the valence band. The events depicted in the figure are as follows. (a) An incoming photon with energy $h v_{1}$ greater than the band gap energy $E_{g}$ is absorbed, creating an EHP; (b) the excited electron gives up energy to the lattice by scattering until it nears the bottom of the conduction band; (c) electron and hole in the conduction and valence bands are moving in the applied field to conduct electric current. An electron in the conduction band can be (d) directly recombined with a hole in the valence band by emitting photon of energy $h v_{2}$, approximately equal to the energy band gap, $E_{g}$ or (e) can be captured by the localized centre by emitting a photon of energy $h v_{3}$ which is less than $E_{g}$. An electron captured by the localized state (f) can be recombined with a hole in the valence band by emitting a photon of energy $h v_{4}$ which is less than $E_{g}$ or $(\mathrm{g})$ can be re-emitted thermally or optically to the conduction band to conduct electric current [11].

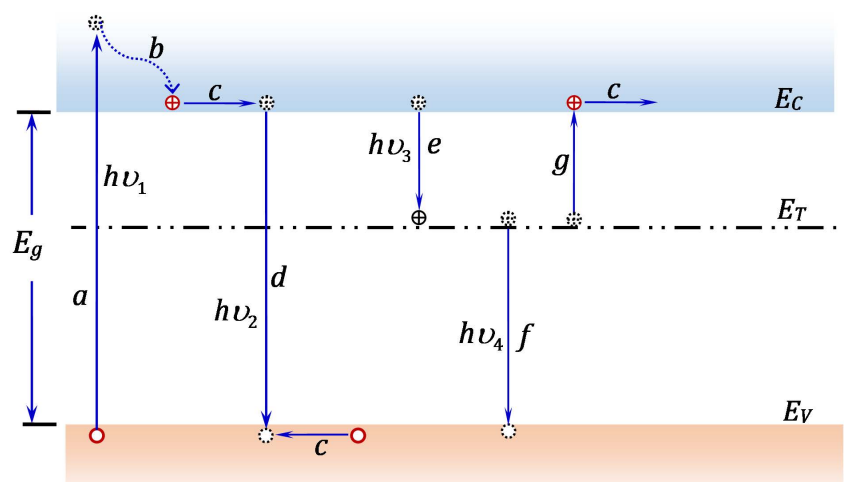

Figure 1: Excitation and recombination mechanisms in photoluminescence with a trapping

In these work, upon the determination of the steady-state expressions for the occupation of free carriers at different energy levels, the relation between excess carrier concentrations under different low injection level is described. Then, by using illumination and net radiative recombination rate the luminescence intensity of light is determined for the corresponding radiative recombination mechanisms by assuming one photon ejects one electron at a time. Finally, by substituting the typical values for energy band gap, capture coefficients, acceptor energy levels and carrier's concentrations of the zinc oxide semiconductor, the competition between the photoluminescence intensity of different localized states are described. This can be done by varying the doping level, energy of the illumination, injection level (incident photo flux density) and temperature of the samples.

In this study, the competition between different localized states and their involvement in photoluminescence intensities of Zinc Oxide compound semiconductor is described. The effects of doping, injection level, temperature and the energy level of the localized states on the photoluminescence of Zinc Oxide are studied.

The rest of this work is organized into four sections. Section two describes the theoretical formulation of photoluminescence intensity and density of free charge carriers in intraband transition state. Section three deals with the numerical result and discussion of effects of photoluminescence intensity on localized state energy and transition energy of zinc oxide compound semiconductor. Section four gives the conclusion.

\section{The photoluminescence intensity and density of free charge carriers in dif- ferent radiative recombination mech- anisms}

Let us assume the localized states are spatially distributed throughout the volume of the semiconductors. The total density of the trap levels occupied by electrons, $n_{T}$ and holes, $p_{T}$ must equal to the total density of traps $N_{T}$ [12].

$$
N_{T}=n_{T}+p_{T}=n_{0 T}+p_{0 T} \quad \text { and } \quad \delta n_{T}=-\delta p_{T}
$$

where, $n_{0 T}$ and $p_{0 T}$ are thermal equilibrium density of the trap levels occupied by electrons and holes respectively.

For the concentrations of photo-generated electrons $(\delta n)$ and holes in the conduction band $(\delta p)$, the respective changes of the concentrations of $p_{T}$ and $n_{T}$ states of a centre due to illumination, $\delta n_{T}$ and $\delta p_{T}$ are given by:

$$
\delta n_{T}=n_{T}+p_{0 T} \quad \text { and } \quad \delta p_{T}=p_{T}+n_{0 T},
$$

where, $\delta n_{T}$ and $\delta p_{T}$ are excess carriers concentrations of electrons and holes on the trap level respectively.

The total concentrations of excess electrons $(\delta n)$ and holes $(\delta p)$ under conduction in the respective conduction and valence bands are then given by:

$$
\delta n=\delta N-\delta n_{T}, \quad \delta p=\delta P-\delta p_{T}
$$

where, $\delta N$ density of electrons and $\delta P$ densities of holes pairs generated in the conduction and valence bands, respectively in a semiconductor due to illumination 
The distribution probability $f_{n}$ for the electron occupation of a trap located at energy $E_{T}$ in the band gap is given by:

$$
f_{n}=\frac{1}{1+\exp \left(\frac{E_{T}-E_{F}}{K_{B} T}\right)}
$$

The thermal equilibrium electron occupancy $n_{0 T}$ and holes occupancy $p_{0 T}$ of the localized states are given by:

$$
n_{0 T}=f_{n} N_{T} \quad \text { and } \quad p_{0 T}=N_{T}-n_{0 T}
$$

The probability of emission of an electron $\left(U_{e n}\right)$ depends only on the electron emission rate $\left(e_{n}\right)$ and the density of traps occupied by electrons $\left(n_{0 T}\right)$. Thus, one can write $U_{c n}$ and $U_{e n}$ by using the principle of detailed balance $\left(R_{t h}=G_{t h}\right)$ as [13]:

$$
\begin{gathered}
U_{c n}=C_{n T} n_{0} p_{0 T}, \quad U_{e n}=e_{n} n_{0 T} \\
U_{c p}=C_{p T} p_{0} n_{0 T} \quad \text { and } \quad U_{e p}=e_{p} p_{0 T} \\
U_{c n}=U_{e n} \quad \text { and } \quad U_{c p}=U_{e p} \\
e_{n}=C_{n T} n_{1} \quad \text { and } \quad e_{p}=C_{p T} p_{1}
\end{gathered}
$$

where,

$$
n_{1}=n_{i} \exp \left(\frac{E_{T}-E_{i}}{K_{B} T}\right) \quad \text { and } \quad p_{1}=n_{i} \exp \left(\frac{E_{i}-E_{T}}{K_{B} T}\right)
$$

where $e_{p}$ is the hole emission rate, $n_{1}$ and $p_{1}$ are the concentrations of electrons and holes in the conduction and valence bands for the case in which the Fermi-level $E_{F}$ falls at $E_{T}$.

The fraction of photo-generated electrons and holes, $I_{n}$ and $I_{p}$ is respectively given by:

$I_{n}=\frac{\delta n_{T}}{\delta n}=\frac{\tau_{p 0} p_{0 T}-\tau_{n 0} n_{0 T}}{\tau_{n 0}\left(p_{0}+p_{1}+n_{0 T}+\delta N\right)+\tau_{p 0}\left(n_{0}+n_{1}+\delta N\right)}$

$I_{p}=\frac{\delta p_{T}}{\delta p}=\frac{\tau_{n 0} n_{0 T}-\tau_{p 0} p_{0 T}}{\tau_{n 0}\left(p_{0}+p_{1}+\delta P\right)+\tau_{p 0}\left(n_{0}+n_{1}+p_{0 T}+\delta P\right)}$

where $\delta N$ is the total excess electron concentration generated in the conduction band and $\delta P$ is the total excess hole concentration generated in the valence band. The subscripts $n$ and $p$ represent the electrons and holes concentration.

\section{Low injection level condition}

Under low injection level system, $N_{T} \gg \delta N, \delta P, \quad p_{0} \gg$ $\delta N, \delta P, p_{1} \gg \delta N, \delta P, n_{1} \gg \delta N, \delta P$,

By using this assumption, $I_{n}$ and $I_{p}$ becomes:

$$
I_{n}=\frac{\tau_{p 0} p_{0 T}-\tau_{n 0} n_{0 T}}{\tau_{n 0}\left(p_{0}+p_{1}+n_{0 T}\right)+\tau_{p 0}\left(n_{0}+n_{1}\right)}
$$

and

$$
I_{p}=\frac{\tau_{n 0} n_{0 T}-\tau_{p 0} p_{0 T}}{\tau_{n 0}\left(p_{0}+p_{1}\right)+\tau_{p 0}\left(n_{0}+n_{1}+p_{0 T}\right)}
$$

where $\tau_{n 0}$ and $\tau_{p 0}$ are constants for a given center and is in general a function of the electron and hole capture coefficients of the center.

\section{Photoluminescence intensity in different Radiative recombination mechanisms}

The total band-to-band radiative recombination rate $R$ is [14].

$$
R=C_{R} n p
$$

where $C_{R}$ is a constant known as the coefficient of holes in the valence band for the capture of electrons from the conduction band and given by [15]:

$$
C_{R}=e \pi \sqrt{k_{\infty}}\left(\frac{m_{n}}{m_{n}{ }^{*}+m_{p}{ }^{*}} \frac{300}{T}\right)^{\frac{3}{2}}\left(1+\frac{m_{n}}{m_{n}^{*}}+\frac{m_{n}}{m_{p}^{*}}\right)\left[E_{g}^{2}\right]
$$

where $e$ is the elemental charge and $k_{\infty}$, the high frequency dielectric constant of the material.

At thermal equilibrium, band-to-band radiative recombination rates are given by:

$$
R^{t h}=C_{R} n_{0} p_{0}
$$

The net band-to-band radiative recombination rate, $U_{R}$ is:

$$
U_{R}=R-R^{t h}=C_{R}\left(p_{0} \delta n+n_{0} \delta p+\delta p \delta n\right)
$$

The ratio of the band to band recombination rate, $U_{R}$ to the free carrier generation rate, $G_{0}$ is given by:

$$
I_{C V}=\frac{U_{R}}{G_{0}}=\frac{C_{R}\left(p_{0} \delta n+n_{0} \delta p+\delta n \delta p\right)}{G_{0}}
$$

The conduction band to localized center radiative recombination rate $R_{C T}$ is:

$$
R_{C T}=C_{n T} n p_{T}
$$

where $C_{n T}$ is a constant known as the coefficient of holes in the localized state for the capture of electrons in conduction band is given by [16]:

$$
C_{C T}=0.69 \times 10^{-12} \frac{\mu}{Z^{2}}\left(\frac{m_{n}}{m_{n}^{*}}\right)^{\frac{5}{2}}\left(\frac{300}{T}\right)^{\frac{1}{2}}\left[E_{C}-E_{T}\right]
$$

The recombination mechanisms in (20) can be rewritten at thermal equilibrium as:

$$
R_{C T}^{t h}=C_{C T} n_{0} p_{0 T}
$$

where, $R_{C T}^{t h}$ is thermal equilibrium conduction band to trap level radiative recombination rate.

The net conduction band to localized trap center radiative recombination rate of free carriers are given by:

$$
U_{C T}=R_{C T}-R_{C T}^{t h}=C_{C T}\left(p_{0 T} \delta n+n_{0} \delta p_{T}+\delta p_{T} \delta n\right)
$$

Or

$$
U_{C T}=C_{C T}\left(p_{0 T} \delta n+n_{0} I_{p} \delta p+I_{p} \delta p \delta n\right)
$$

The ration of the conduction band recombination rate of free carriers to the total optical generation rate of the free carriers, $G_{0}$ is given by:

$$
I_{C T}=\frac{U_{C T}}{G_{0}}=\frac{C_{C T}\left(p_{0 T} \delta n+n_{0} I_{p} \delta p+I_{p} \delta p \delta n\right)}{G_{0}}
$$


The localized state to valence band radiative recombination rate $R_{T V}$ is :

$$
R_{T V}=C_{T V} p n_{T}
$$

where, $C_{T V}$ is a constant known as the coefficient of holes in the valence band for the capture of electrons in the localized states and it is given by [17].

$$
C_{T V}=0.69 \times 10^{-12} \frac{\mu}{Z^{2}}\left(m_{n} / m_{p}^{*}\right)^{5 / 2}(300 / T)^{1 / 2}\left(E_{T}-E_{V}\right)
$$

The recombination mechanisms in (26) at thermal equilibrium, this recombination rate is given by:

$$
R_{T V}^{t h}=C_{T V} p_{0} n_{0 T}
$$

The net localized trap center to valence band radiative recombination rate of free carriers are:

$$
U_{T V}=R_{V}-R_{V}^{t h}=C_{T V}\left(p_{0} \delta n_{T}+n_{0 T} \delta p+\delta p \delta n_{T}\right)
$$

Or

$$
U_{T V}=C_{T V}\left(p_{0} I_{n} \delta n+n_{0 T} \delta p+I_{n} \delta p \delta n\right)
$$

The intensity of light from the trap level to the valence band, $I_{T V}$ is given by:

$$
I_{T V}=\frac{U_{T V}}{G_{0}}=\frac{C_{T V}\left(n_{0 T} \delta p+p_{0} I_{n} \delta n+I_{n} \delta p \delta n\right)}{G_{0}}
$$

\section{Numerical Result and Discussion}

In this topic the factors that affect photoluminescence intensities of the free carrier's density and the factors that affect the intensities of the lights emitted between different energy levels in $\mathrm{ZnO}$ semiconductor is described in detail.

\subsection{Variation of photoluminescence intensity of Zinc Oxide with localized state energy}

In this section, the localized energy dependence of the photoluminescence intensities of Zinc Oxide are described at different temperatures. The photoluminescence intensities for the conduction band to valence band, $I_{C V}$, conduction band to localized states $I_{C T}$, and for the localized states to valence band $I_{T V}$ transitions are described using relations (19), (25) and (31) above respectively.

Figure 2 illustrates the variation of low injection level photoluminescence intensities due to various electronic transition levels with localized energies at (a) $60 \mathrm{~K}$, (b) $500 \mathrm{~K}$ and (c) $900 \mathrm{~K}$ for zinc oxide compound semiconductor. As depicted in Figure 2 (a), the conduction band to valence band photoluminescence $I_{C V}$ is very high at the low energy regimes because of the absence of free electrons trap by the low energy localized states. However, the conduction band to valence band photoluminescence $I_{C V}$ is decreasing with increasing the energy of the localized states above the mid-gap due to the small free electrons density in the conduction band due to the increase in the free electrons trap by the localized states. The conduction band to valence band photoluminescence $I_{C V}$ increases in the donor region towards the valence band because of the reduction (absence) of free electrons trap by the localized states.

At very low temperature, most of the localized states are occupied by the trapped free carriers and hence there is no (very small) recombination taking place between the conduction band and the localized state; and between the localized states and the valence band as shown in Figure 2 (a). As the temperature increases, the free carriers trapping effect by the localized states decreases. This facilitates the radiative recombination rates between all the energy levels. As a result, the photoluminescence due to the transition of free electrons from the conduction band to the valence band $I_{C V}$, from the conduction band to the localized states $I_{C T}$ and from the localized states to the valence band $I_{T V}$ are increasing as shown in Figure 2 (a) to (c).
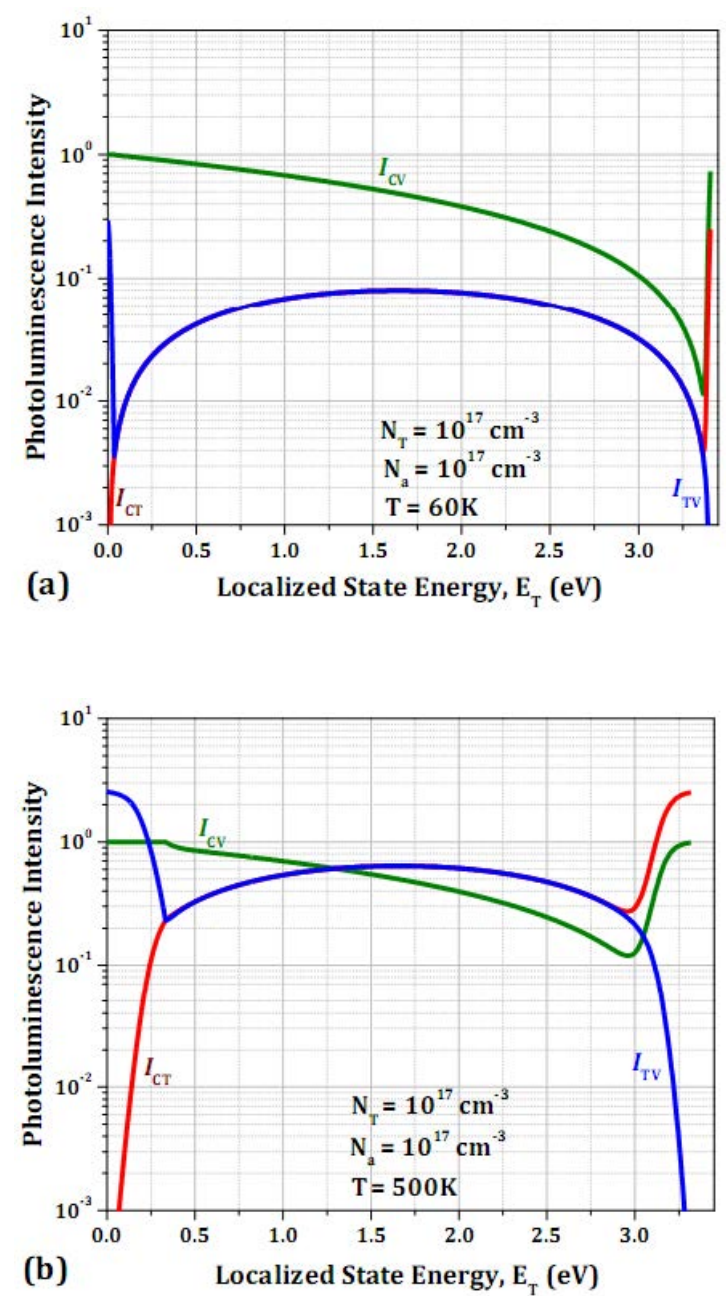

All the localized states above the acceptor level and below the donor level have equal probability for the recombination of free electrons in the conduction band with the available holes in the localized states; and free electrons in the localized states with holes in the valence band. Since they are in the same electronic states, there is no transition of free electrons from 


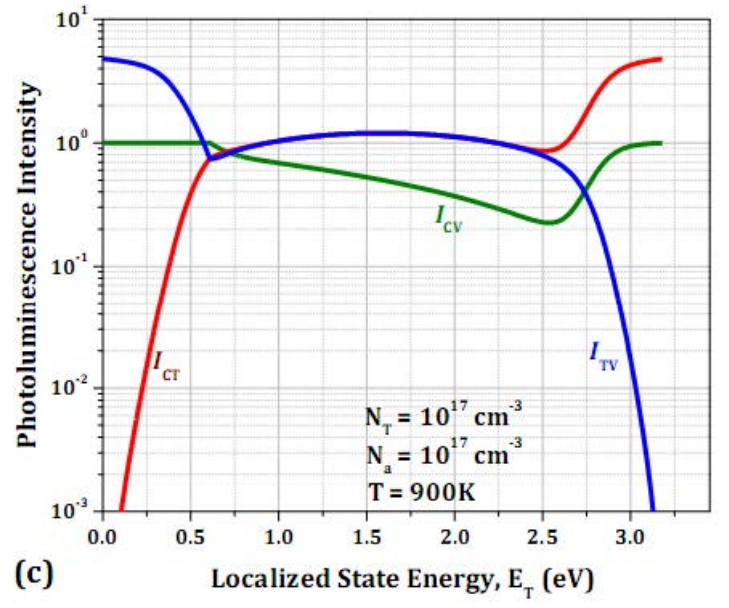

Figure 2: Variation of low injection level photoluminescence intensities due to various electronic transition levels with localized energies at (a) $60 \mathrm{~K}$, (b) $500 \mathrm{~K}$ and (c) $900 \mathrm{~K}$ for zinc oxide

the conduction band to the acceptor levels; and from the donor level to the valence band. However, there is very high degree of electron transition (photoluminescence) from conduction band to donor levels; and from acceptor levels to the valence band.

Note that, the band to band photoluminescence intensity dominates at very low temperature and the localized state to valence band photoluminescence intensity dominates at very high temperature (Figure 2 (a) and (c)). At the intermediate temperature, the band to band photoluminescence dominates the lower energy regimes and the localized states to the valence bands photoluminescence dominates the higher energy regimes as depicted in Figure 2 (b). The acceptor level to valence band and the valence band to donor level free electron transition rates are the most dominant at very high temperature as depicted in Figure 2(c).

\subsection{Variation of photoluminescence intensities of zinc oxide with transition energy}

The conduction band to valence band photoluminescence intensity is described only by a single transition energy $E_{C}-E_{V}$ at constant temperature. The conduction band to localized state photoluminescence intensity is described using the variation in $E_{C}-E_{T}$. The localized state to valence band photoluminescence intensity is described using the variation in $E_{T}-E_{V}$.

Figure 3 illustrates the variation of low injection level photoluminescence intensities due to various electronic transition levels with transition energies at (a) $60 \mathrm{~K}$, (b) $500 \mathrm{~K}$ and (c) $900 \mathrm{~K}$ for the sample used in Figure 2. Please, refer to the description of Figure 2 for more detail of the effects of localized energy states the photoluminescence intensities of different transition levels. As it can be seen in Figure 3 (a) to (c), the conduction band to valence band photoluminescence under goes only a single transition energy of $E_{C}-E_{V}$ at constant temperature. This energy transition level is relatively shifted to low energy level with increasing temperature, since the band gap is decreasing with increasing temperature. In conduction band to the localized state transition of free carriers, $E_{C}-E_{T}$ is considered as low transition energy for $E_{T}$ above the midgap and vice versa. Hence, in the description of the photoluminescence intensities as a function of $E_{C}-E_{T}$, the red graphs of $I_{C T}$ in Figure 2 above have to be inverted horizontally as shown in Figure 3. In the localized state to valence band transition of free carriers, $E_{T}-E_{V}$ is considered as low transition energy for $E_{T}$ below the mid-gap and vice versa. Hence, in the description of the photoluminescence intensities as function of $E_{T}-E_{V}$, the blue graphs of $I_{T V}$ in Figure 2 above have to be remained as they are as shown in Figure 3.
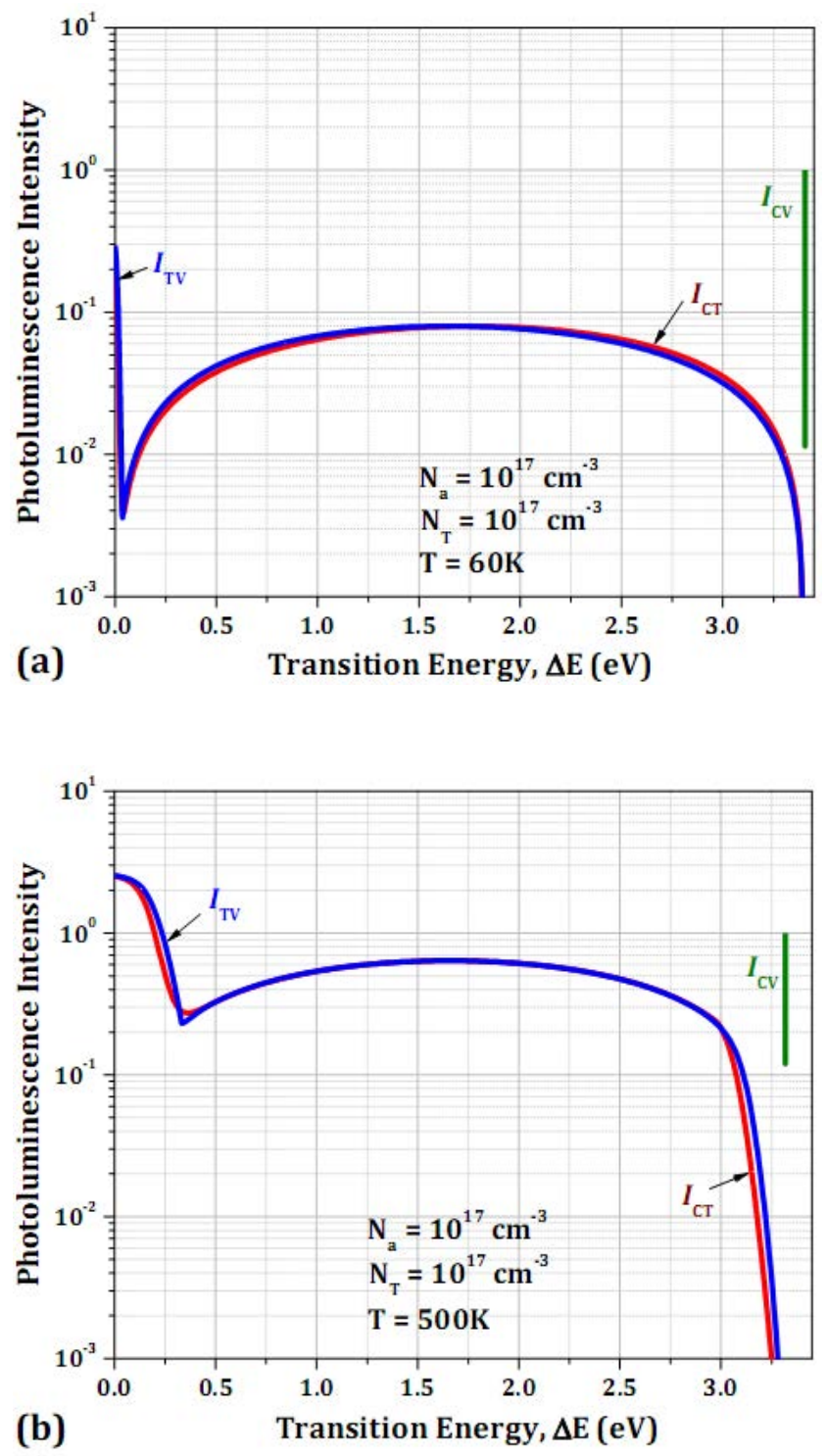


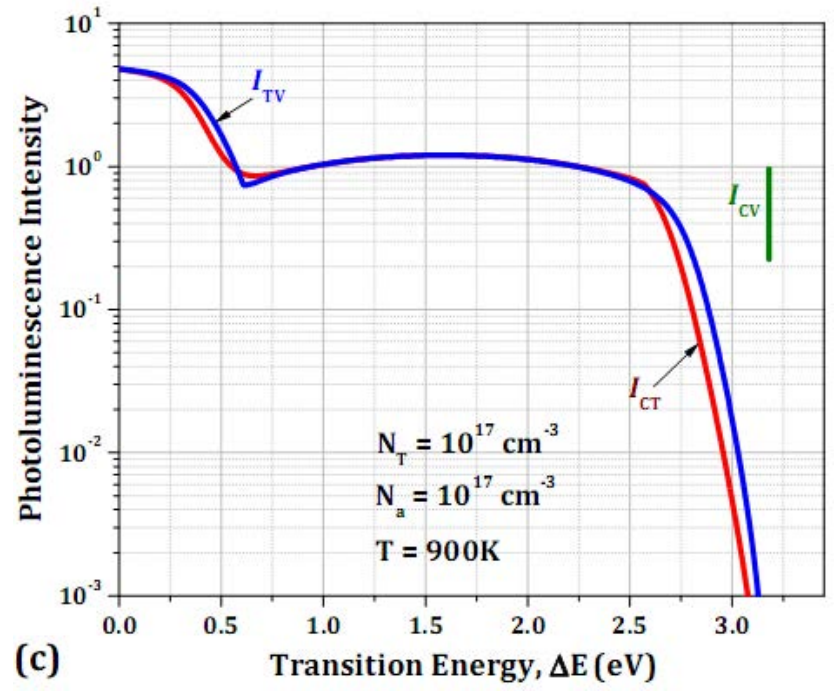

Figure 3: Variation of low injection level photoluminescence intensities due to various electronic transition levels with transition energies at (a) $60 \mathrm{~K}$, (b) $500 \mathrm{~K}$ and (c) $900 \mathrm{~K}$ for the sample used in zinc oxide

Hence, except the reduction of the lower and higher transition energies conduction band to localized photoluminescence, $I_{C T}$ depicted in Figure 3 (b) and (c), the variation of $I_{C T}$ and $I_{T V}$ with transition energy is similar at the intermediate transition energies at all temperatures. The variations of high temperature $I_{C T}$ and $I_{T V}$ at the lower and higher transition energies are attributed to the high intensity of the capture of free electrons in the deep donor levels in low energy transition regimes and due to the highly ionized acceptor levels in the high transition energy regimes. The increase in the minimum value of $I_{C V}$ with increasing temperature depicted in Figure 3 (a) to (c) is due to the reduction of carrier trapping.

One can conclude from Figure 3 that, if an intensive photoluminescence is observed closer to band edge, its source could be most probably band to band free carrier transition, but not localized state band free carrier transition. The other photoluminescence intensities observed at transition energies far below the band edge energy could be attributed to the conduction band to localized state or localized state to valence band free carrier transitions.

\section{Conclusion}

This work has discussed the photoluminescence of zinc oxide compound semiconductor. The intensity of light depends on the amount of illumination and net rate of recombination of excess electrons in the corresponding radiative recombination mechanisms. It shows the amount of light in the bands and the more luminescent bands. Since there is no effects of traps in the conduction to valence band radiative recombination, rate of recombination is high and photoluminescence intensity of light becomes high. The intensity of light decreases with increasing localized trap energy in this band. It was shown that due to trap effects in the conduction band to trap level radia- tive recombination, the recombination rate reduces and hence decreases the intensity of light in the band. The intensity of light increases with increasing localized trap energy in this band. Due to the effects of trap, only small amount of electrons on localized Centre and excess of holes in the valence band. These decreases the recombination rates and hence decreases the intensity of light in the Trap level to valence band radiative recombination. It has been shown that, the trapping intensities varies with temperature. At low temperatures, free carrier traps are relatively more active. The minority carrier traps farther away above the intrinsic level become more effective with decreasing temperature. Free carrier traps are more effective at low temperatures, when compared to high temperatures. The probability of emission of carriers by the center increases with increasing temperature. The electron trap localized centers become the most dominant electron trap centers at lower temperatures.

Photoluminescence is varied with localized state energy and transition energy at different temperatures. Under variation with localized state energy, at very low temperature, there is no (very small) recombination taking place between the conduction band and the localized state; and between the localized states and the valence band. As the temperature increases, the photoluminescence due to the transition of free electrons from the conduction band to the valence band, $I_{C V}$, from the conduction band to the localized states, $I_{C T}$ and from the localized states to the valence band, $I_{T V}$ are increasing.

The band to band photoluminescence intensity dominates at very low temperature and the localized state to valence band photoluminescence intensity dominates at very high temperature. At the intermediate temperature, the band to band photoluminescence dominates at the lower energy regimes and the localized states to the bands photoluminescence dominates the higher energy regimes. The acceptor level to valence band and the valence band to donor level free electron transition rates are the most dominant at very high temperature.

It was concluded that, under variation of Photoluminescence with transition energy, the conduction band to valence band photoluminescence energy transition level is relatively shifted to low energy level with increasing temperature. the variation of $I_{C T}$ and $I_{T V}$ with transition energy is similar at the intermediate transition energies at all temperatures.

\section{Acknowledgements}

I would like to thanks my family for their various supports during the preparation of this manuscript.

\section{REFERENCES}

[1] Smith W. "Curious effect of Light on Selenium", . s.1. : Scientific American, ,(29 March 1873).

[2] Ogale S B. Thin Films and Heterostructures for Oxide Electronics, New York: Springer, (2005). 
[3] S. Yamauchi, Y. Goto, and T. Hariu, Photoluminescence studies of undoped and nitrogen-doped $\mathrm{ZnO}$ layers grown by plasma-assisted epitaxy, J. Cryst. Growth 260 1-6, (2004).

[4] E. K. Ellmer, A. Klein, and B. Rech, Transparent Conductive Zink Oxide Springer, Berlin, (2008).

[5] E. V. Kortunova, N. G. Nikolaeva, P. P. Chvanski, et al., J. Mater. Sci. 43, 2336 (2008).

[6] S.M. Sze, Physics of Semiconductor Devices, John Wiley and Sons, New York, (1981).

[7] Nickel N H and Terukov E (ed) Zinc Oxide-A Material for Micro- and Optoelectronic Applications Netherlands: Springer, (2005).

[8] S. Rein, Lifetime spectroscopy: A method of defect characterization in silicon for photovoltaic applications, Springer, Berlin, Germany (2004).

[9] W. W. Moses, Nucl. Instrum. Methods Phys. Res. A 487, 123 (2002). 5. S. H. Wei and A. Zunger, Appl. Phys. Lett. 72, 2011 (1998).

[10] D. W. Hamby, D. A. Lucca, M. J. Klopfstein and G. Cantwell, Temperature dependent exciton photoluminescence of bulk ZnO. J. Appl. Phys. 3214-3217 93 (2003).

[11] A. Hangleter, R. Hacker, Enhancement of band-toband Auger recombination by electron-hole concentration, Phys. Rev. Lett. 65 (2), 215-18, (1990).

[12] W. Shockley, W. Read, Statistics of the recombinations of holes and electrons, Physical Rev.87 835842. (1952).

[13] J. J. Sumakeris, and M. J. O’Loughlin, Appl. Phys. Lett. 88, 052110, (2006).

[14] H. Matsunami and T. Kimoto, Material Science and Enginering, R. 20, 125 (1997).

[15] Charina L Choi, Kristie J Koski, Sanjeevi Sivasankar, and A Paul Alivisatos. Strain Dependent Photoluminescence Behavior of CdSe / CdS Nanocrystals with Spherical Linear, and Branched Topologies. Nano Letters, 9(10):3544-3549, (2009).

[16] Dunlap.R.A, "Expermental Physics," in Modern Method, New York, years,(1988).

[17] Gebrehiwet.H, "Absorption Coefficient and Dielectric Function of Direct Band Gap Silicon Nanocrystallities," pp,(200-204) years, (2006). 